\title{
Réacteur VUV à décharge interne pour le dépôt en couches minces de silicium amorphe, d'oxyde et de nitrure de silicium
}

\author{
C. Fuchs, R. Henck et E. Fogarassy
}

Centre de Recherches Nucleaires (CRN), Laboratoire PHASE (UPR du CNRS n²92), BP. 20, 67037 Strasbourg Cedex 2, France

\begin{abstract}
We have developed a new VUV $(100<\lambda<200 \mathrm{~nm})$ photo-enhanced chemical vapor deposition (Photo-CVD) technique, using an internal hydrogen discharge lamp. By this way the need for a window between the incident excitation light and reactant gas $\left(\mathrm{SiH}_{4}, \mathrm{Si}_{2} \mathrm{H}_{6}, \mathrm{O}_{2}, \mathrm{~N}_{2} \mathrm{O}, \mathrm{N}_{2}, \mathrm{NH}_{3}\right)$, is eliminated thus overcoming the problems of attenuation of short wavelength radiation by the window for two reasons : a limited transparency of the available optical materials in the VUV range and a progressive window opacification by film deposition during the process. Possibilities given by this reactor, for depositing large area hydrogenated amorphous silicon and silicon nitride are demonstrated.
\end{abstract}

\section{Introduction}

Le développement de nouvelles techniques photoassistées de dépôts en couches minces des matériaux électroniques représente un enjeu technologique majeur. En effet, elles permettent de réaliser, à basse température, des films de nombreux métaux, isolants et semiconducteurs, en dissociant avec une source de lumière ultraviolette les molécules d'un gaz (ou mélange gazeux) approprié par un processus photolytique [1]. Les atomes ou radicaux photogénérés sont susceptibles de diffuser dans la phase gazeuse pour se condenser sur le substrat du dépôt. Ces expériences sont généralement effectuées au moyen de sources de lumière (lasers ou lampes) situées à l'extérieur de la chambre de réaction, configuration qui présente, cependant, deux inconvénients majeurs. $D^{\prime}$ une part, on observe, durant le processus, une opacification progressive de la fenêtre d'entrée du réacteur par dépôt de matière sur sa face interne, ce qui a pour conséquence de stopper les mécanismes photodissociatifs responsables de la croissance du film. D'autre part, les optiques de transmission dont on dispose dans le domaine ultra-violet $(\lambda<200 \mathrm{~nm})$ ont une transparence limitée aux longueurs d'onde les plus courtes (165 nm pour le quartz suprasil, 115 et $105 \mathrm{~nm}$ pour $\mathrm{MgF}_{2}$ et $\mathrm{LiF}$, respectivement). Notons par ailleurs que les radiations UV intenses ont des effets secondaires néfastes sur ces optiques.

Ces difficultés nous ont conduit à nous intéresser à un concept nouveau [2], consistant à produire le rayonnement ultra-violet directement dans le milieu réactif au moyen $d$ 'une décharge dans l'hydrogène.

\section{Dispositif expérimental - dépôts en couches minces}

La chambre de réaction est équipée, en plus d'un porte-substrat chauffant et d'une rampe à injection des gaz, $\mathrm{d}^{\text {un }}$ système de décharge constitué d'une paire d'électrodes en acier-inox refroidies par circulation d'eau ou en silicium de basse résistivité, alimentées à basse fréquence $(50 \mathrm{~Hz})$.

Des mesures directes de l'émission VUV produite par une décharge dans l'hydrogène ( $3 \mathrm{mbar}$ ) ont été effectuées au moyen d'un spectromètre fonctionnant dans l'UV lointain $(\lambda \geq 115 \mathrm{~nm})$. Elles nous ont permis d'obtenir le spectre de l'émission (Fig. 1) qui se caractérise par la présence de deux bandes centrées autour 
des longueurs d'onde 120 et $160 \mathrm{~nm}$, respectivement. Il faut toutetois noter que cette émission a été enregistrée en présence d'hydrogène seul. Nous avons observé que l'introduction de gaz réactifs dans l'enceinte conduisait à des modifications de l'émission VUV de la figure 1. Ce spectre recouvre parfaitement les spectres d'absorption des différents gaz réactifs utilisés pour l'élaboration de films de Si-a $(\mathrm{H})$, $\mathrm{SiO}_{2}$ et $\mathrm{Si}_{3} \mathrm{~N}_{4}$ (Fig. 2). Les énergies des photons dont on dispose sont telles que la photodissociation de $\mathrm{SiH}_{4}$ en $\mathrm{SiH}_{3}, \mathrm{SiH}_{2}$ et $\mathrm{SiH}$ et de $\mathrm{Si}_{2} \mathrm{H}_{6}$ en $\mathrm{SiH}_{3}, \mathrm{Si}_{2} \mathrm{H}_{5}, \mathrm{Si}_{2} \mathrm{H}_{4}$ et $\mathrm{Si}_{2} \mathrm{H}_{3}$ est possible. L'ensemble de ces radicaux devrait participer à la croissance des films et feront l'objet d'investigations futures.

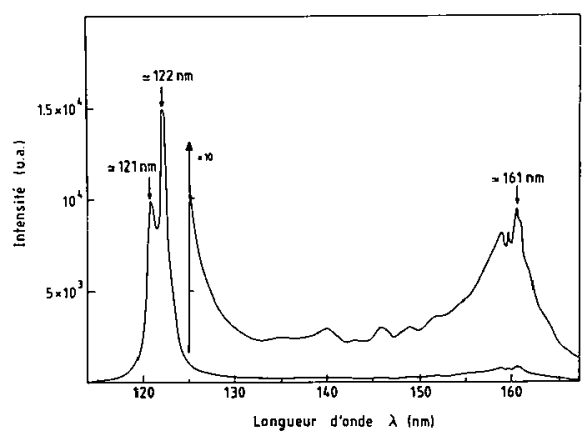

Fig. 1 - Spectre d'émission d'une décharge dans $\mathrm{H}_{2}(\mathrm{p}=3$ mbar $)$

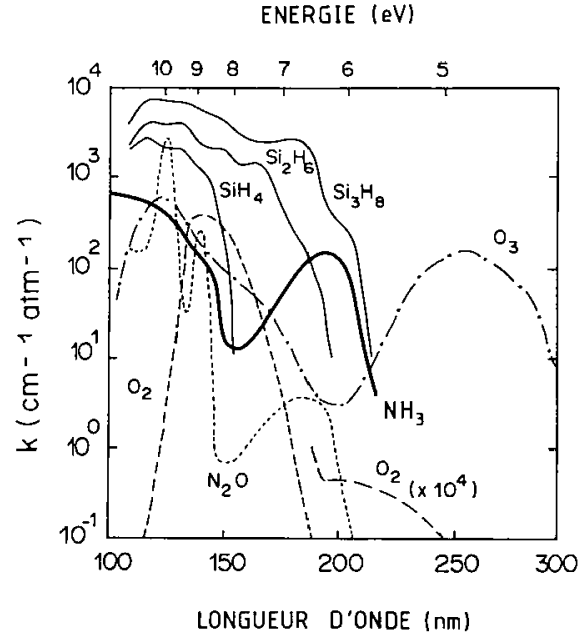

Fig. 2 - Spectres d'absorption de différents gaz dans le domaine UV

Nous nous sommes attachés à optimiser d'une part la rampe d'injection des gaz actifs et de l'hydrogène, d'autre part la géométrie et la nature des électrodes, pour répondre à deux impératifs : (a) fournir un rayonnement uniforme sur de grandes surfaces $\left(\varnothing \approx 4^{\prime \prime}\right)$; (b) éliminer ou réduire le bombardement des gaz réactifs et du substrat de dépôt par les ions et les électrons de la décharge afin, d'une part, de limiter les processus dissociatifs par choc, d'autre part prévenir la dégradation des films durant leur croissance.

Cette technique nous permet, à l'heure actuelle, de réaliser des dépôts de silicium amorphe hydrogéné et de nitrure de silicium (jusqu'à $1 \mu \mathrm{m}$ d'épaisseur) avec des vitesses de croissance de 10 à $100 \AA /$ minute, vitesses comparables à celles observées dans les réacteurs à plasma ou obtenues par d'autres techniques photoassistées [1].

\section{Références}

[1] "Photochemical Processing of Electronic Materials", edited by BOYD I.W. and JACKMAN R.B. (Academic Press, London, 1992).

[2] MILNE W.I., CLOUGH F.J., DEANE S.C., BAKER S.D., ROBERTSON P.A., Appl. Surf. Sc. 43 (1989) 277. 